\title{
THE HYDROGEN ABUNDANCES IN WN STARS
}

\author{
W.-R. HAMANN ${ }^{1}$, G. DÜNNEBEIL ${ }^{1}$, U. WESSOLOWSKI ${ }^{1}$, W. SCHMUTZ ${ }^{2}$ \\ ${ }^{1}$ Institut für Theoretische Physik und Sternwarte der Universität, \\ Olshausenstraße 40, D-2300 Kiel, Federal Republic of Germany \\ 2 Joint Institute for Laboratory Astrophysics, University of Colorado \\ Boulder, Colorado 80309-0440, USA
}

Hydrogen abundances in WN stars have been derived by Conti et al. (1983) from a "semiquantitative" study of the Balmer-Pickering decrement. No clear correlation between hydrogen detection and spectral subtype could be established: stars with hydrogen and stars without detectable hydrogen are found among both, the WNE ("early") as well as the WNL ("late").

This picture drastically changes when the hydrogen detection is not correlated with the subtype, but instead with the stellar parameters as obtained by a detailed spectral analyses with our elaborate models (Schmutz et al., 1989). Now we find a clear separation between two groups: the "cool" stars with $T_{*} \approx$ $35 \mathrm{kK}$ exhibit hydrogen, while the "hot" stars (T. > $60 \mathrm{kK}$ ) do not. WR136 is intermediate in temperature and in hydrogen abundance. Some of those stars classified as "early" subtypes are actually "cool", and just these stars are found to show hydrogen.

We now apply our model calculations for a precise analysis of hydrogen abundances. For that purpose we extended our model code for a fully consistent treatment of line blends. We perform a "fine analysis" of the helium spectrum, giving special weight to the fit of those He II Pickering lines which are not blended by hydrogen. Subsequently, the theoretical profiles of those He II lines which coincide with hydrogen Balmer lines are compared with the observation. The best fit yields the stellar parameters ( $R$., $T_{*}, \dot{M}, v_{\infty}$ ) and the hydrogen mass fraction, $B_{H}$ (Table 1 ).

The hydrogen mass fractions obtained for WR40 and WR128 $\left(B_{H} \approx 20 \%\right)$ agree well with theoretical predictions for a post-RSG evolution. WR136 with its smaller value $\left(B_{H}=6 \%\right)$ appears as a link to the "hot" WN stars in which hydrogen is not detectable. WR22 is either in pre-RSG stage or represents the result of binary evolution.

Table 1. Fine Analyses Including Hydrogen

\begin{tabular}{lcccc}
\hline & WR 40 & WR 128 & WR 22 & WR 136 \\
\hline Subtype & WN8 & WN4 & WN7abs & WN6 \\
T./kK & 31.2 & 37 & 31 & 51 \\
$R_{\odot} / R_{\odot}$ & 18. & 5.3 & 30 & 6.0 \\
$\log \left\{\dot{M} /\left(\mathrm{M}_{\odot} \mathrm{yr}^{-1}\right)\right\}$ & -4.05 & -5.0 & 4.32 & -3.85 \\
$v_{\infty} /(\mathrm{km} / \mathrm{s})$ & 1000 & 2000 & 1500 & 1700 \\
$\log \mathrm{L} / \mathrm{L}_{\odot}$ & 5.4 & 4.7 & 5.9 & 5.5 \\
$B_{\mathrm{H}}[\%]$ & $16 \pm 5$ & $20 \pm 10$ & $40 \pm 5$ & $6 \pm 3$ \\
\hline
\end{tabular}

\section{References}

Conti, P.S., Leep, E.M., Perry, D.N.: 1983, Ap. J. 268, 228

Schmutz, W., Hamann, W.-R., Wessolowski, U.: 1989, Astr. Ap. 210, 236

$K$. A. van der Hucht and B. Hidayat (eds.),

Wolf-Rayet Stars and Interrelations with Other Massive Stars in Galaxies, 100.

(c) 1991 IAU. Printed in the Netherlands. 